\title{
Construyendo el capital intelectual en la gestión del conocimiento para el aprendizaje en una administración pública española Building the intellectual capital in the Knowledge Management for learning in a Spanish Public Administration
}

\author{
Luis Martín-Fernandez ${ }^{1}$, Margarita Martínez-Núñez², Waldo S. Pérez-Aguiar² ${ }^{2}$ Maria Pilar Latorre Martinez ${ }^{3}$, Ramón \\ Hermoso $^{4}$ \\ LMFernandez@mapama.es,margarita.martinez@upm.es, waldo.perez@upm.es, latorrep@unizar.es, rhermoso@unizar.es
}

${ }^{1}$ D.G. Desarrollo Rural y Politica Forestal

Ministerio de Agricultura y Pesca, Alimentación y Medio Ambiente Madrid, España
${ }^{2}$ Centro de Investigación en Tecnologías Software y Sistemas Multimedia para la sostenibilidad (CITSEM)

Universidad Politécnica de Madrid

Madrid, España

\author{
${ }^{3}$ Departamento de \\ Dirección y Organización \\ de Empresas
}

Universidad de Zaragoza

Zaragoza, España
${ }^{4}$ Departamento de Informática
e Ingeniería de Sistemas

Universidad de Zaragoza

Zaragoza, España

\begin{abstract}
Resumen- Las organizaciones públicas son grandes productoras y consumidoras de conocimiento. La Gestión del Conocimiento y su utilización inteligente permiten generar valor público en sus actuaciones. El conocimiento es el conjunto de nociones y habilidades por las cuales los miembros de una organización pública atienden y completan su trabajo. Uno de los primeros pasos hacia una Gestión Inteligente del Conocimiento en una Administración Pública Forestal es la construcción y definición del capital intelectual mediante la socialización del conocimiento en el que se muestra el "saber hacer" de la organización. Esta comunicación presenta los resultados y conclusiones del Curso "Gestión y Política Forestal en la Administración General del Estado en España” como una experiencia innovadora y pionera de construcción del capital intelectual en la Administración Forestal del Estado dentro de un sistema de Gestión del Conocimiento para el aprendizaje. Por último, se indican los siguientes pasos y factores a tener en cuenta para la valoración del capital intelectual en una Administración Pública Forestal dentro de Sistema de Gestión del Conocimiento.
\end{abstract}

Palabras clave: Gestión del conocimiento, administración publica, capital intelectual, gestión y política forestal

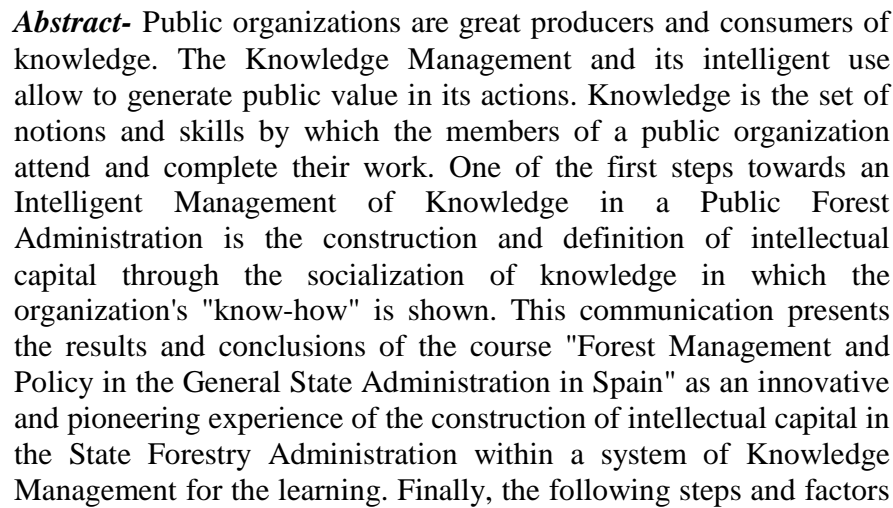

to be taken into account for the valuation of intellectual capital in a Public Forest Administration within the Knowledge Management System

Keywords: Knowledge management, public administration, intellectual capital, Forest management and policy

\section{INTRODUCCIÓN}

El conocimiento es fundamental para la formulación de políticas y los servicios públicos (Blackman et al., 2013 y Kim y Lee, 2006 ). El conocimiento es el conjunto de nociones y habilidades (Bloom, 1970) por las cuales los miembros de una organización pública atienden y completan su trabajo. El conocimiento es dinámico, está en continua evolución, adquiere su valor cuando es usado en un tiempo y lugar específico. Sin un contexto especifico seria información, no conocimiento, (Nonaka y Konno, 1.998).

El sector público produce y difunde grandes cantidades de información (Prokopiadou et al, 2004). Toda esa información debe ser adecuadamente gestionada para generar conocimiento, y a través del conocimiento, crear valor público para la sociedad. Kebede (2010) define el concepto de gestión del conocimiento como una gestión decidida y sistemática del conocimiento y los procesos y herramientas asociados a éste con el objetivo de conseguir explotar plenamente su potencial $\mathrm{y}$ que, de esta forma, sirva de apoyo a las decisiones y resolución de problemas, facilitando las innovaciones y la creatividad dentro de la organización.

El Marco Estratégico para la FAO 2000-2015 refleja claramente la importancia que atribuye la FAO a la gestión de los conocimientos como forma de ayudar y apoyar a sus Estados Miembros. En el sector forestal en general y en la FAO en particular, es importante compartir los conocimientos entre todos los interesados. Existe un reto global de la gestión 
de los conocimientos forestales para todos los implicados en el sector forestal. El Comité Forestal de la FAO invita a los miembros de la organización y al sector forestal en su conjunto a apoyar las actividades de gestión de los conocimientos que permitan desarrollar y compartir conocimientos relativos a la actividad forestal.

Uno de los primeros pasos hacia una Gestión del Conocimiento en una Administración Pública Forestal es la construcción y definición del capital intelectual mediante la socialización del conocimiento en el que se muestra el "saber hacer” de la organización. En España todavía no existe una clara apuesta por este tema, aunque comienzan a tomar conciencia y a implementarse distintas iniciativas a nivel sectorial. En esta comunicación se estudia este tipo de iniciativa y se selecciona y analiza un caso precursor de este tipo de iniciativas de sociabilización del conocimiento.

El objetivo de esta comunicación es presentar los avances en la definición del capital intelectual de una administración pública forestal, a través de una experiencia de socialización del conocimiento que se está generando en la Administración Forestal del Estado.

La iniciativa seleccionada se basa en el curso "Gestión y Política Forestal en la Administración General del Estado” que se celebró en 2016 y estuvo enfocado como una experiencia para socializar el conocimiento y la actividad que se desarrolla en la Administración Forestal del Estado. Esta comunicación describe los resultados y conclusiones de este curso desde el punto de vista de la gestión del conocimiento. Por último, se indican los siguientes pasos y factores a tener en cuenta para la valoración del capital intelectual de una Administración Pública Forestal en el marco de un Sistema de Gestión del Conocimiento.

\section{CONTEXTO}

La gestión del conocimiento, a partir de un conjunto de procesos y sistemas, busca que el capital intelectual de una organización aumente de forma significativa (Nuñez, 2003). Una adecuada Gestión del Conocimiento, adaptada a las peculiaridades propias de las Administraciones Públicas, permitirá transformar el conocimiento individual de algunos funcionarios en un activo para la Administración (Troncoso, 2000). Gestionar el conocimiento implica por lo tanto identificar y analizar el conocimiento disponible en una organización (Llorca, 2002). El concepto de Capital Intelectual se define como la acumulación de conocimiento que crea valor o riqueza cognitiva poseída por una organización, compuesta por un conjunto de activos de naturaleza intangible o recursos y capacidades basados en conocimiento (Bueno et al, 2008).

La Administración Pública proporciona un contexto idóneo para analizar la gestión del conocimiento, dado que se compone de un conjunto heterogéneo de organismos públicos que van desde centros de investigación hasta empresas públicas de diversa índole, permitiendo al analista obtener una visión general de cómo el conocimiento se crea, transforma, distribuye y evoluciona en contextos ricos y heterogéneos bajo el paraguas de lo público, de tal forma que posteriormente dichos resultados obtenidos puedan aplicarse para mejorar los ecosistemas tecnológicos en cualquier ámbito de actuación mediante la extensión del know-how y la sabiduría sobre el dominio del problema obtenida en este tipo de contextos (Rowley, 2007).

El aprendizaje se convierte en un pilar dentro de cualquier tipo de organización, independientemente de su tamaño o de la actividad que desarrolle. Desde las Pequeñas y Medianas Empresas (PYME) hasta instituciones como las Universidades o la Administración Pública (AP), incorporan dentro de sus procesos mecanismos que permiten la gestión del conocimiento (García-Holgado et al, 2015).

\section{DESCRIPCIÓN}

Para alcanzar los objetivos propuestos para esta comunicación, se ha seguido el Modelo de Creación de Conocimiento de Nonaka y Takeuchi (1995) en el que existen cuatro pasos fundamentales para la creación de conocimiento: socialización, externalización, combinación e internalización.

Dentro del modelo seguido, la socialización es un proceso de compartir experiencias y, a través de ellas, crear tanto conocimiento como habilidades técnicas y conocimientos compartidos. Según Nonaka y Konno (1998), la fase de socialización en el proceso de creación de conocimiento consiste en que se compartan experiencias y modelos de trabajo, eliminando así las barreras entre cada miembro de la organización y los demás miembros.

Esta metodología fue aplicada en el curso “Gestión y Política Forestal en la Administración General del Estado“ durante los meses de Abril - Mayo 2016, organizado por la Dirección General de Desarrollo Rural y Política Forestal del Ministerio de Agricultura, Alimentación y Medio Ambiente. Los objetivos del mismo eran dar a conocer las actividades que realiza la Administración General del Estado en España en materia de Gestión y Política Forestal y fomentar el intercambio de experiencias y conocimientos entre profesionales forestales.

El curso tuvo 100 participantes vinculados con el sector forestal que desarrollan su actividad profesional en distintas administraciones públicas (estatal, autonómica y local), en organismos públicos de investigación y docencia, en empresas privadas o que querían aumentar su formación y conocimiento de las actividades en gestión y política forestal del Estado.

Se contó con 30 funcionarios del Ministerio de Agricultura, Alimentación y Medio Ambiente, como instructores. Desarrollan su trabajo en todos los ámbitos de la actividad forestal que se llevan a cabo en el Ministerio de Agricultura, Alimentación y Medio Ambiente. Son precisamente, estos 30 funcionarios, los que se han seleccionado como muestra y por tanto, sobre los que se ha aplicado esta experiencia de socialización del conocimiento forestal generado en el Ministerio.

El curso de "Gestión y Política Forestal en la Administración General del Estado" tuvo una duración de 70 h. Se impartió en la modalidad semipresencial con sesiones presencial y retransmitidas online y grabadas, y una plataforma Moodle de apoyo docente. El curso se celebró en los meses de Abril - Mayo 2016, fue organizado por la Dirección General de Desarrollo Rural y Politica Forestal del Ministerio de Agricultura, Alimentación y Medio Ambiente, dentro del Programa de Formación para el Desarrollo Rural 2016. Este curso tuvo 100 participantes con interés en la actividad forestal del Ministerio y 30 profesores, todos ellos 
funcionarios del Ministerio de Agricultura, Alimentación y Medio Ambiente.

El curso se estructuró en los módulos que se indican en la Tabla 1:

Tabla 1.

Módulos del Curso de "Gestión y Política Forestal en la Administración General del Estado"

Módulo 1: Competencias forestales en la estructura de la

Administración General del Estado

Ley de Montes. Comités nacionales.

Módulo 2: Política Forestal Internacional

Estrategia Forestal Europea y Plan de Acción Forestal Europeo. Criterios e

Indicadores de Gestión Forestal Sostenible. FOREST EUROPE. Otros foros internacionales

Módulo 3: Planificación y Legislación Foresta

Estrategia y Planes Nacionales. Directrices básicas comunes. Montes de Utilidad Pública

Módulo 4: Información y Estadística Forestal

Inventario Forestal Nacional. Mapa Forestal. Redes Europeas de Seguimiento de Bosques. Estadística Forestal.

Módulo 5. Conservación y protección de los montes

- $\quad$ Sanidad Foresta

- Incendios Forestales

- $\quad$ Restauración Forestal: Inventario Nacional de Erosión de Suelos

Módulo 6: Recursos Genéticos Forestales

Mejora y conservación de recursos genéticos forestales. Estrategia Española.

Red de Centros. Producción y comercialización de los materiales forestales de reproducción

Módulo 7: Medidas Forestales en Programas de Desarrollo Rural (PDR)

Reglamento FEADER. MEC y otros fondos estructurales. Marco Naciona de Desarrollo Rural. Desarrollo y medidas forestales cofinanciadas en los PDR.

\begin{tabular}{c}
\hline Módulo 8: Caza y Pesca. Aprovechamientos forestales no madereros \\
\hline $\begin{array}{c}\text { Módulo 9: La gestión forestal en el Dominio Público Hidráulico. } \\
\text { Restauración ambiental de ecosistemas fluviales degradados }\end{array}$ \\
\hline Módulo 10. Normativa para la comercialización de productos de la \\
madera aprovechados legalmente \\
Reglamentos EUTR y FLEGT \\
Módulo 11: La gestión forestal en espacios protegidos \\
Parques Nacionales \\
Red Natura 2000
\end{tabular}

Cada módulo contaba con el contenido didáctico que se muestra en la Figura 1:

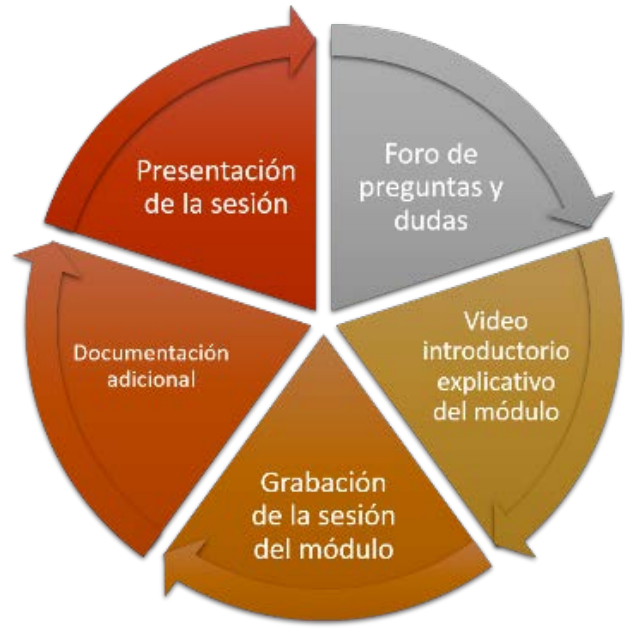

Figura 1. Contenido didáctico de cada módulo del Curso de "Gestión y Politica Forestal en la Administracion General del Estado
Como se puede observar en la figura 1, cada uno de los módulos estuvo diseñado para que existieran distintos canales de información, cada uno de ellos en distintos soportes siendo las sesiones virtuales y el foro, los entornos donde se genera una mayor socialización entre los distintos participantes e instructores. Por tanto, las nuevas tecnologías vinculadas al diseño de los módulos dan origen a entornos de socialización combinados según las distintas actividades desarrolladas.

\section{RESUltados}

La socialización del conocimiento es el proceso de adquirir conocimiento tácito a través de compartir experiencia por medio de exposiciones, documentos, manuales y formas de actuación que añade el conocimiento a la base colectiva que posee la organización (Nonaka y Takeuchi 1995). En este estudio piloto entendemos por socializar el conocimiento identificar aquellas acciones participativas donde se comparta, interaccione y se colabore, dentro de una base colectiva como es la plataforma Moodle.

Antes de presentar los resultados se indica la caracterización de la muestra. La selección inicial de los 30 instructores funcionarios del Ministerio de Agricultura, Alimentación y Medio Ambiente es la siguiente (Tabla 2):

Tabla 2.- Caracterización de la muestra

\begin{tabular}{|l|c|r|}
\hline \multicolumn{3}{|c|}{ Sexo } \\
\hline Hombres & 22 & $73,33 \%$ \\
\hline Mujeres & 8 & $26,67 \%$ \\
\hline Edad \\
\hline 2: Entre 40 - 50 & 8 & $40,00 \%$ \\
\hline 3: Menos de 40 & 10 & $26,67 \%$ \\
\hline
\end{tabular}

Las acciones participativas de donde se han obtenido los resultados de sociabilización son las sesiones virtuales y los foros. En ambos casos, los instructores mediante documentos y mensajes, visualizaban, compartían, interaccionaban con los conocimientos de otros instructores a través de la plataforma:

\section{Las sesiones virtuales}

Sesiones virtuales generalmente incluyen distintas oportunidades de sociabilización en línea ( Hofmann, 2003 ). En el caso de estudio, como se observa en la figura 1, cada módulo presentaba la posibilidad de trabajar en línea con las sesiones en dos modalidades, asíncrona y síncrona. La forma de trabajar de manera asíncrona se realiza mediante los videos de presentación y la grabación de las distintas sesiones.

La configuración en línea de manera sincrónica se lleva a cabo dentro de las sesiones virtuales mediante las salas de chat, conferencias de audio y vídeo, la pantalla y uso compartido de aplicaciones. Actualmente, son consideradas como una de las grandes fuentes de participación, colaboración e interacción (Çardak y Selvi 2016) y por consiguiente de socialización. 
La mayoría de las actividades de las sesiones puede ser fácilmente llevada a cabo en una sesión virtual con la ayuda de las herramientas tecnológicas necesarias. En nuestro caso de estudio, las presentaciones de las sesiones fueron grabadas en plataforma Moodle mediante la herramienta BlackboardColaborate. Estas sesiones virtuales eran accesibles a través de un enlace directo desde la página web relacionada con el curso. Los instructores y participantes podían enviar mensajes escritos, generándose tres tipos de interacción: instructorparticipante, instructor-instructor y participante-participante.

\section{Los foros}

Los foros son entornos de aprendizaje en línea asíncronos donde instructores y participantes interactúan juntos. Estos están abiertos durante todo el día, y dan soporte a acciones de carácter cooperativo a través de discusiones. En este sentido, los foros mediante los mensajes permiten que la interacción sea posible en cualquier lugar, cualquier situación, y en cualquier momento (Dawley, 2007). Las actividades que se pueden organizar en una plataforma tipo foro incluyen entradas en las que los instructores y participantes pueden compartir sus opiniones, conocimientos y experiencias (Karaman, 2009). En consecuencia, las actividades de discusión y estudios cooperativos pueden llevarse a cabo a través de diferentes técnicas (Kalelioğlu y Gülbahar, 2010).

\section{Nivel de socialización (segmentación de las acciones)}

Podemos definir distintos niveles de sociabilización. La visualización tanto de documentación como de mensajes de todo el conocimiento puede considerarse un paso anterior a la interacción en entornos vinculados a otros instructores.

En ambas acciones participativas los mensajes son un vehículo clave para compartir experiencias y conocimientos pero la capacidad de los instructores en distintos módulos del que se le ha invitado a participar de forma directa, es considerada como un elemento clave en la sociabilización.

En la Figura 2, se comparan tres vías de interacción de los mensajes: acciones en entornos directos: la visualización de los mensajes, la generación de mensajes y acciones en nuevos entornos participativos: generación de mensajes en foros y sesiones virtuales distintos a los propios.

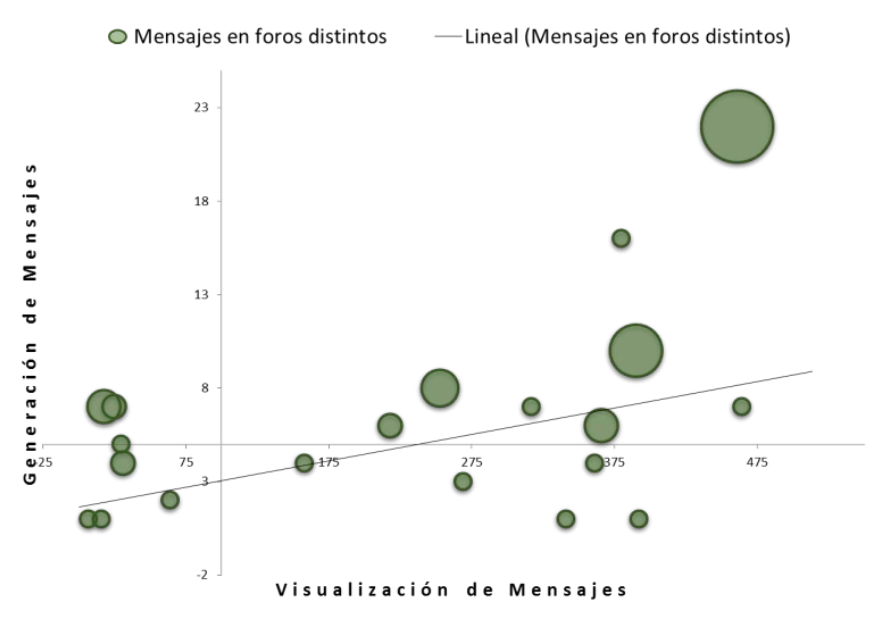

Figura 2.-Conocimiento tácito: Actividades de interacción y colaboración mediante mensajes

Como se puede apreciar en la Figura 2, existe una clara relación entre la generación de mensajes y la participación en entornos diferentes al propio, por lo que el nivel de sociabilización está muy vinculado a una actitud activa de los instructores frente a las visualizaciones.

A la hora de compartir conocimiento mediante documentación sin la existencia de la interacción entre individuos, se observa que la disposición a visualizar y adquirir documentación en entornos distintos a los propios es muy baja. También se distingue en la figura 3, una correlación entre aquellos individuos más activos con la visualización de documentos de su propio entorno son aquellos capaces de transitar a otros entornos externos al suyo.

Por último cabe destacar que la interacción entre individuos a través de mensajes proporciona un mayor nivel de actividad socializadora frente a las actividades de visualización de la documentación.

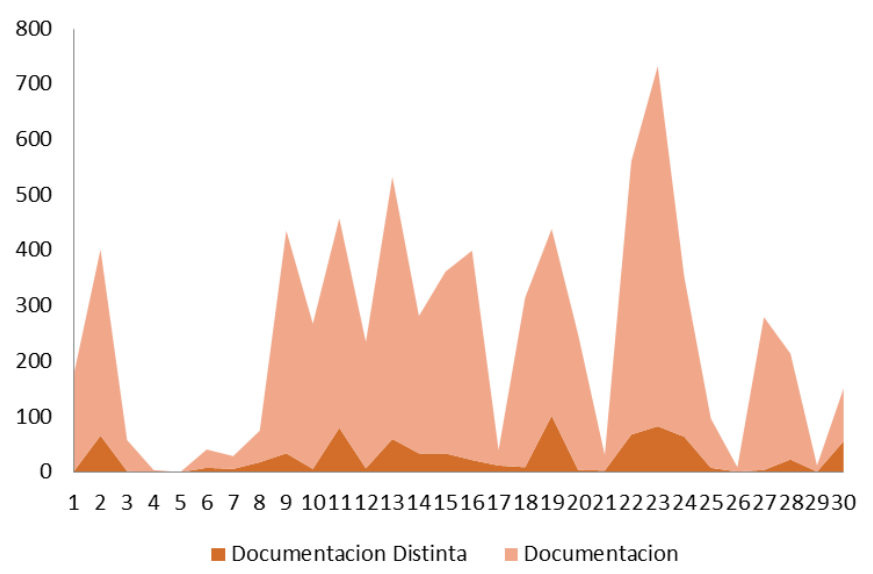

Figura 3.- Conocimiento tácito: Visualización de documentación y manuales

\section{Patrones de sociabilización de los instructores}

Otro punto a tener en cuenta es el análisis de los patrones de comportamiento de los instructores a través de las actividades llevadas a cabo en las cinco configuraciones diferentes vinculadas a las sesiones virtuales y a los foros (Tabla 3).

Tabla 3.- Patrones de comportamiento: Análisis por rango de edad

\begin{tabular}{|c|l|r|r|r|}
\cline { 2 - 5 } \multicolumn{1}{c|}{} & \multicolumn{1}{c|}{ Población } & $\begin{array}{c}\text { Casos } \\
(\mathrm{N})\end{array}$ & Media & Desv. Tip \\
\hline \multirow{4}{*}{$\begin{array}{c}\text { Vistas } \\
\text { documentación }\end{array}$} & Total & 30 & 214,20 & 174,48 \\
\cline { 2 - 5 } & 1: Mas de 50 & 12 & 147,08 & 146,57 \\
\cline { 2 - 5 } & 2: Entre 40 - 50 & 8 & 285,00 & 194,39 \\
\cline { 2 - 5 } & 3: Menos de 40 & 10 & 238,10 & 177,07 \\
\hline \multirow{4}{*}{$\begin{array}{c}\text { Vistas } \\
\text { Mensajes }\end{array}$} & Total & 30 & 185,47 & 180,37 \\
\cline { 2 - 5 } & 1: Mas de 50 & 12 & 167,25 & 193,63 \\
\cline { 2 - 5 } & 2: Entre 40 - 50 & 8 & 219,63 & 174,28 \\
\cline { 2 - 5 } & 3: Menos de 40 & 10 & 180,00 & 184,04 \\
\hline $\begin{array}{c}\text { Actividad } \\
\text { Mensajes en }\end{array}$ & Total & 30 & 4,27 & 5,02 \\
\cline { 2 - 5 } & 1: Mas de 50 & 12 & 4,08 & 4,64 \\
\hline
\end{tabular}




\begin{tabular}{|c|l|r|r|r|}
\multirow{4}{*}{ foros } & 2: Entre $40-50$ & 8 & 5,13 & 7,38 \\
\cline { 2 - 5 } & 3: Menos de 40 & 10 & 3,80 & 3,39 \\
\hline \multirow{4}{*}{$\begin{array}{c}\text { Actividad } \\
\text { Mensajes en } \\
\text { foros distintos }\end{array}$} & Total & 30 & 2,00 & 3,81 \\
\cline { 2 - 5 } & 1: Mas de 50 & 12 & 1,08 & 1,51 \\
\cline { 2 - 5 } & 2: Entre $40-50$ & 8 & 3,75 & 6,34 \\
\cline { 2 - 5 } & 3: Menos de 40 & 10 & 1,70 & 2,98 \\
\hline \multirow{4}{*}{$\begin{array}{c}\text { Documentación } \\
\text { Distinta }\end{array}$} & Total & 30 & 27,23 & 30,28 \\
\cline { 2 - 5 } & 1: Mas de 50 & 12 & 15,42 & 21,84 \\
\cline { 2 - 5 } & 2: Entre 40 - 50 & 8 & 35,50 & 36,68 \\
\cline { 2 - 5 } & 3: Menos de 40 & 10 & 34,80 & 31,84 \\
\hline
\end{tabular}

Como se puede observar en la Tabla 3 en las 5 configuraciones los instructores con una edad comprendida entre los 40-50 años son los que mayor actividad generan, produciéndose en este segmento también las mayores variaciones de actividad. En el caso de análisis por género (Tabla 4) el comportamiento de los hombres es más activo a excepción de una de las actividades de socialización con mayor repercusión como es la interacción mediante la visualización de documentación distinta al entorno propio. Esto puede ser debido a la diferencia entre las actividades síncronas donde el hombre tiene un patrón más activo, frente a esta última donde el momento de consulta no es necesario que sea en un tiempo determinado, sino que su disponibilidad en el tiempo es mayor.

Tabla 4.- Patrones de comportamiento: Análisis por género

\begin{tabular}{|c|c|c|c|c|c|}
\hline & Población & $\begin{array}{c}\text { Casos } \\
(\mathrm{N})\end{array}$ & Media & $\begin{array}{l}\text { Media } \\
\text { Norm. }\end{array}$ & $\begin{array}{l}\text { Desv. } \\
\text { Tip }\end{array}$ \\
\hline \multirow{3}{*}{$\begin{array}{c}\text { Vistas } \\
\text { Documentación }\end{array}$} & Total & 30 & 214,20 & 214,20 & 174,48 \\
\hline & Hombre & 22 & 227,05 & 309,61 & 179,11 \\
\hline & Mujer & 8 & 178,88 & 670,80 & 167,14 \\
\hline \multirow{3}{*}{ Vistas Mensajes } & Total & 30 & 185,47 & 185,47 & 180,37 \\
\hline & Hombre & 22 & 208,95 & 284,93 & 179,43 \\
\hline & Mujer & 8 & 120,88 & 453,30 & 178,04 \\
\hline \multirow{3}{*}{$\begin{array}{l}\text { Actividad } \\
\text { Mensajes en } \\
\text { foros }\end{array}$} & Total & 30 & 4,27 & 4,27 & 5,02 \\
\hline & Hombre & 22 & 5,14 & 7,01 & 5,46 \\
\hline & Mujer & 8 & 1,88 & 7,05 & 2,42 \\
\hline \multirow{3}{*}{$\begin{array}{c}\text { Actividad } \\
\text { Mensajes en } \\
\text { foros distintos }\end{array}$} & Total & 30 & 2,00 & 2,00 & 3,81 \\
\hline & Hombre & 22 & 2,46 & 3,35 & 4,37 \\
\hline & Mujer & 8 & 0,75 & 2,81 & 0,71 \\
\hline \multirow{3}{*}{$\begin{array}{l}\text { documentación } \\
\text { Distinta }\end{array}$} & Total & 30 & 27,23 & 27,23 & 30,28 \\
\hline & Hombre & 22 & 26,23 & 35,77 & 31,58 \\
\hline & Mujer & 8 & 30,00 & 112,50 & 28,19 \\
\hline
\end{tabular}

\section{CONCLUSIONES}

Las organizaciones públicas son grandes productoras, generadoras y distribuidoras de conocimiento. Todo ese conocimiento debe ser adecuadamente gestionado para, a través del conocimiento, crear valor público para la sociedad.

La experiencia de socialización del conocimiento presentada en esta comunicación es un primer paso hacia una Gestión del Conocimiento en una Administración Pública Forestal. La construcción y definición del capital intelectual mediante la socialización del conocimiento muestra el "saber hacer” de la organización.

Este trabajo presenta como caso de estudio el curso "Gestión y Política Forestal en la Administración General del Estado” que se celebró en 2016 y estuvo enfocado como una experiencia para socializar el conocimiento. Para alcanzar los objetivos propuestos para esta comunicación, se ha seguido el Modelo de Creación de Conocimiento de Nonaka y Takeuchi (1995)

Del análisis de la actividad de los instructores en el curso, se concluye que las acciones participativas donde se han obtenido los resultados de sociabilización son las sesiones virtuales y los foros. Los patrones de visualización e interacción revelan que, las distintas actividades combinadas podrían servir de factores para el diseño de futuras prácticas para mejorar la socialización de conocimiento en una administración pública.

En los niveles de socialización analizados cabe destacar que la interacción entre individuos a través de mensajes proporciona un mayor nivel de actividad socializadora frente a las actividades de visualización de la documentación.

Esta experiencia de socialización del conocimiento analizada en esta comunicación es un punto de partida para la construcción del capital intelectual de una Administración Pública Forestal. 


\section{REFERENCIAS}

Blackman, D., Kennedy, M., Burford, S., \& Ferguson, S. (2013). Introduction to the special symposium on knowledge management and public administration: Good bedfellows or potential sparring partners. International Journal of Public Administration, 36(3), pp 151-154.

Bloom, B. S. (1977). Taxonomía de los objetivos de la educación. El Ateneo.

Bueno, E., Salmador, M. P., \& Merino, C. (2008). Génesis, concepto y desarrollo del capital intelectual en la economía del conocimiento: Una reflexión sobre el Modelo Intellectus y sus aplicaciones. Estudios de economía aplicada, 26(2), pp 43-63.

Çardak, Ç. S., \& Selvi, K. (2016). Increasing teacher candidates' ways of interaction and levels of learning through action research in a blended course. Computers in Human Behavior, 61, pp 488-506.

Dawley, L. (Ed.). (2007). The tools for successful online teaching. IGI Global.

Garcia-Hidalgo, A., Cruz-Benito, J., Garcia-Peñalvo, F.J. (2015). Análisis Comparativo de la gestión del conocimiento en la administración pública española. En Fidalgo Blanco, A., Sein-Echaluce Lacleta, M.L., García-Peñalvo, F.J. (2015) La Sociedad del Aprendizaje. Actas del III Congreso Internacional sobre Aprendizaje, Innovación y Competitividad. CINAIC 2015 (14-16 de Octubre de 2015, Madrid, España). Madrid. Fundación General de la Universidad Politécnica de Madrid

Hofmann, J. (2003). The synchronous trainer's survival guide: Facilitating successful live and online courses, meetings, and events. John Wiley \& Sons.

Kalelioglu, F., \& Gulbahar, Y. (2009). Use of social software in education: a multiple intelligences perspective. Educational Social Software for Context-Aware Learning: Collaborative Methods and Human
Interaction: Collaborative Methods and Human Interaction, 50.

Karaman S. (2009). Internet y Redes de Ordenadores y Comunicaciones [redes informáticas y de comunicación] Nobel, Ankara, Cordero (Ed.), pp. 371-426

Kebede, G. (2010). Knowledge management: An information management perspective. International Journal of Information Science, 30, pp 416-424.

Kim, S., \& Lee, H. (2006). The impact of organizational context and information technology on employee knowledge sharing capabilities. Public Administration Review, 66(3), pp 370-385.

Llorca, E. (2002). CEVALSI: un nuevo concepto de observatorio tecnológico para un nuevo modelo de sociedad. Economía industrial, (343), pp 131-146.

Nonaka, I \& Konno, N (1998). The concept of Ba: Building a fundation for knowledge creation. California Management Review, Vol.40 ,no.3, Spring

Nonaka,I. \& Takeuchi,H. (1995).The knowledge-creating company: How Japanese Companies Create the Dynamics of Innovation. Oxford University Press, New York.

Núñez, M. O. (2003). El capital intelectual en la gestión del conocimiento. Revista Cubana de los Profesionales de la Información e la Comunicación en Salud, (6).

Prokopiadou, G., Papatheodorou, C., \& Moschopoulos, D. (2004). Integrating knowledge management tools for government information. Government Information Quarterly, 21(2), pp 170-198.

Rowley, J. (2007). The wisdom hierarchy: representations of the DIKW hierarchy. Journal of information science, 33(2), 163-180. doi: 10.1177/0165551506070706

Troncoso, A. (2000). “Gestión del Conocimiento en las Administraciones Públicas”, en: Gestión del Conocimiento y Capital Intelectual, Instituto Universitario Euroforum Madrid, pp. 35-39. 